Vietnam Academy of Science and Technology
Vietnam Journal of Earth Sciences
Website: http://www.vjs.ac.vn/index.php/jse

\title{
Interpretation of land cover using spectral modulation pattern- an example with Landsat 8 OLIimage
}

\author{
Nguyen Dinh Duong*, Le Van Anh, Ho Le Thu \\ Institute of Geography, Vietnam Academy of Science and Technology, Hanoi, Vietnam
}

Accepted 25 December 2014

\begin{abstract}
Spectral signature, in general, can be defined as characteristics of surface objects of transmission, absorption and reflection of electromagnetic radiation. Spectral signature is expected stable and unique for given surface material. Spectral signature can be graphically represented in two-dimensional space in the form of spectral curves. Spectral signature has been used long time for object detection and classification mostly by spectral matching methods. Spectral matching is time-consuming and requires reference spectra that is not always available in hand. Spectral modulation pattern is a simplified form of spectral curve shapes. We define this pattern by pairwise comparison of reflectance between two spectral bands. The aim of this research is to point out the use of spectral modulation patterns for land cover mapping. The experiment has been carried out with Landsat 8 OLI image data, which has six reflective bands of $30 \mathrm{~m}$ spatial resolution. The Landsat 8 OLI is an excellent data source for land cover mapping in both local and global scale. Due to very huge data volume, automated analysis is crucial when we need accomplish land cover maps in a short time. In this paper, the authors introduce the concept to use spectral modulation patterns of spectral signatures for automated interpretation of land cover. The first step in this concept is to understand correctly meaning of each modulation pattern of spectral signatures and their relation to land cover classes. In this study, we use three Landsat 8 OLI images of Vietnam in 2013 and 2014. Ground GPS field photos were collected to support interpretation of land cover.
\end{abstract}

Keywords: Spectral signature, Spectral reflectance curve, Landsat 8 OLI, modulation pattern, Visual interpretation.

(C) 2014 Vietnam Academy of Science and Technology

\section{Introduction}

Spectral signature is one of the basic concepts of spectral remote sensing from the beginning. Spectral signature defines unique correspondence between the material and its reflectance (Shaw et al., 2003). Two-dimension plot of the spectral signature is spectral reflectance curves (SRC), its shape is formed by varying reflectance amplitude. We hypothesize that spectral reflectance curves of similar features have similar shapes. Therefore, the shape of spectral reflectance curves has been

*Corresponding author, Email: duong.nguyen2007@gmail.com broadly exploited in detection algorithms where more weight is given to the shape than to the amplitude (Shaw et al., 2003).

In general, to detect a ground object, the SRC is to be compared with the one in spectral libraries. The comparison can be done by quantitative or qualitative spectral matching techniques (Thenkabail et al., 2007; Ruby et al., 2002). Obviously, without reference spectra, no matching technique can be executed. Matching is a pixelbased algorithm with complex computation of various variables like spectral correlation similarity, Euclidian distance similarity, spectral 
Nguyen Dinh Duong et al./Vietnam Journal of Earth Sciences 36 (2014)

similarity value or spectral angle similarity, etc. This computation is time-consuming and has to carry out repeatedly for each pixel with all reference spectra in the library. Evidently, if the matching is implemented with little reference spectra only, the analysis process could be much accelerated.

Because the shape of SRC is stable to a land cover type so once, the relation is established, we can use it forever. The SRCis usually expressed in a graphical form like curve, and it is hard to express the relation between SRC and land cover type analytically. There have been few reports on an effort of digital encoding the shape of SRC and its application in land cover mapping. The central thesis of this paper focuses on digital encoding of SRC shape and their interpretation from land cover mapping point of view.

In this paper, the authors develop a method to group spectral signatures into patterns of SRCcurves. These patterns will be used for an explanation of land cover types, which associate with those patterns. The study pointed out that meaning of SRCcurve patterns is stable, and we can use this meaning for quick interpretation of land cover. In this research, we use the six reflective bands of Landsat 8 OLI image data for three regions of Vietnam covering northern and central provinces. Research results were validated by ground GPS field photos.

\section{Method}

\subsection{Study Area}

To illustrate the method we developed here, we use three Landsat 8 OLI images as given in Table 1. The selected three OLI scenes represent typical geographic regions of Vietnam (Figure 3). Land cover of the northern area - scene number LC81270452013336LGN00 is dominated by evergreen vegetation cover, paddy field and developed land (Hanoi City). The other two scenes in the central part of Vietnam cover highland region, which features both evergreen and deciduous forest, industrial tree plantation (coffee, rubber etc.). The scene LC81240522014046LGN00 also includes coastal zone with sandy terrain and semi-arid landscape cover. All the three scenes were acquired during the dry season. Cloud coverage was in its low level, so most of the areas are under clear sky, and ground objects were very well interpreted.

Table 1. Used Landsat 8 OLI scenes

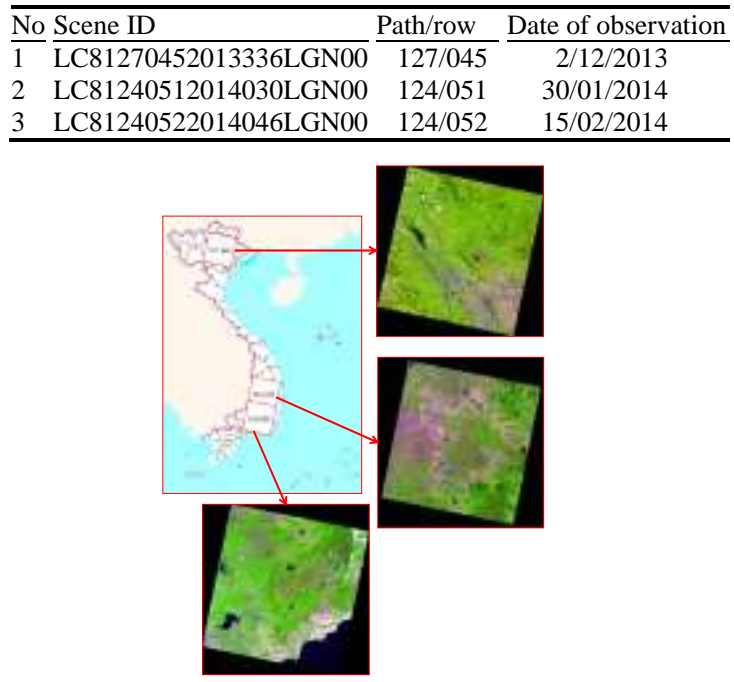

Figure 1. Location of the Landsat 8 OLI scenes over Vietnam

\subsection{SRC Modulation Pattern Concept}

In this research,we use six reflective bands of the OLI sensor, and they are blue, green, red, near infrared, short wave infrared SWIR 1 and short wave infrared SWIR 2.These bands are named, respectively, as band $1,2,3,4,5$ and 6 with abbreviations $b_{1}, b_{2}, b_{3}, b_{4}, b_{5}$ and $b_{6}$. Values of these variables are counted in reflectance by conversion of digital numbers DN to reflectance using gain and offset given in the metadata file of each Landsat OLI scene. Each pixel vector has six values and can be written as $\vec{p}=$ $\left(b_{1}, b_{2}, b_{3}, b_{4}, b_{5}, b_{6}\right)$ where $b_{i}$ are reflectance value in bandi. Spectral remote sensing expects that different surface materials feature different pixel vectors and objects of similar surface material are represented by similar pixel vectors. Twodimensional plot of pixel vector where one axis shows reflectance value and the other displays spectral band number defines so-called spectral reflectance curve SRC. Figure 2 shows an example of spectral reflectance curves of water, vegetation, bare land and cloud. We use here short wave infrared color composite RGB: 5, 4, 3 to plot the SRC. 


\section{Vietnam Journal of Earth Sciences 36 (2014) 480-488}

As seen on Figure 2, different surface objectshave different SRCs. These curves differ in different values of reflectance in each band and result curves with different shapes. It is quite challenging to formulate shape of each reflectance curve in digital form. The authors develop here a system for encoding the spectral reflectance curves. Duong (1997) has reported similar effort when conducted research with ADEOS-II GLI data. The encoding scheme is pairwise and relies on a comparison of relative positions of two vertices. When two vertices have the same position (equal reflectance value), the score is 1 . If the position of a preceding vertex is higher than the position of the other vertex, the score is 2 , and if lower the score is 0 . By this way, each pixel vector can be encoded by a series of digits $0,1,2$ Depending on the relative position among vertices. To encode modulation of a spectral reflectance curve, which has $n$ vertices, we need $\frac{n(n-1)}{2}$ digits. In the caseof Landsat 8 OLI with six reflective bands, we need 15 digits in total. Let us use $m_{i, i+l}$ to denote the position value between vertex $i$ and $i+1$

(a)
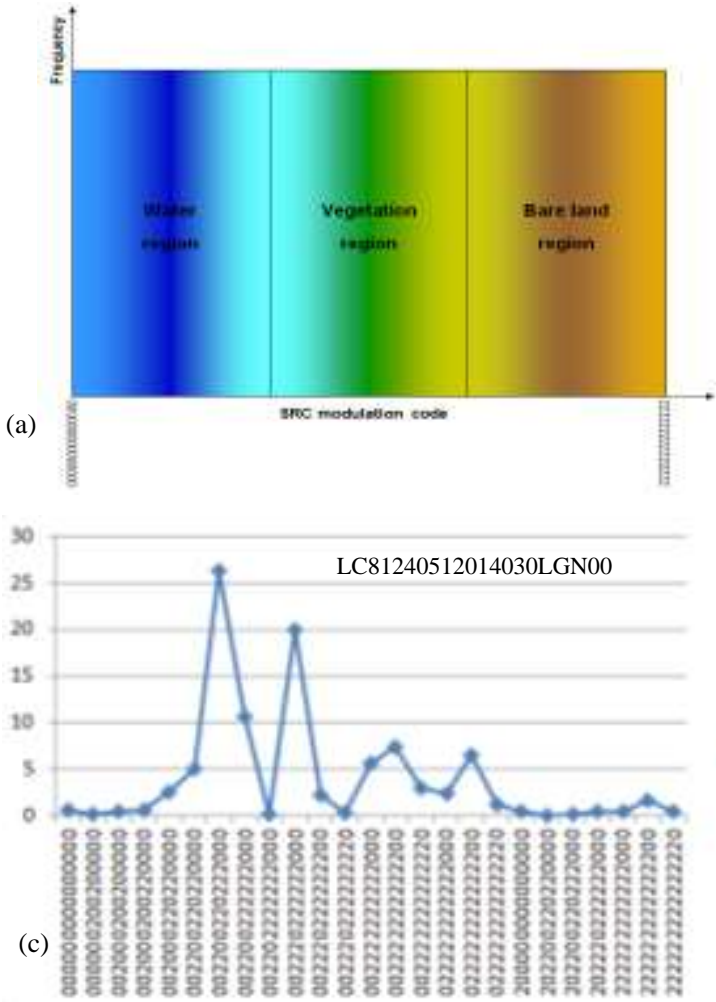

then for Landsat 8 OLIpixel vector we have 15 digits for encoding of SRC modulation.

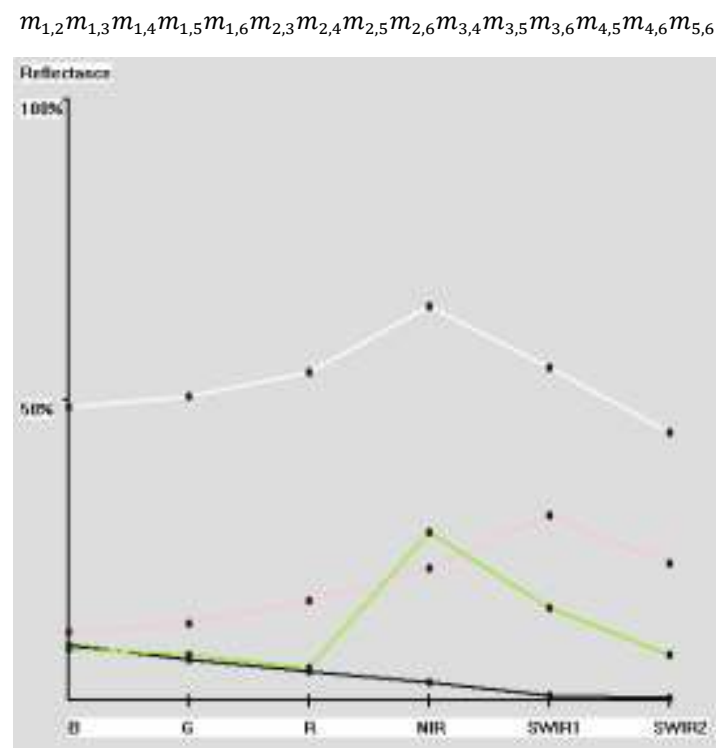

Figure 2. Spectral reflectance curve of cloud (white), barren land (pink), vegetation (green) and water (dark blue)
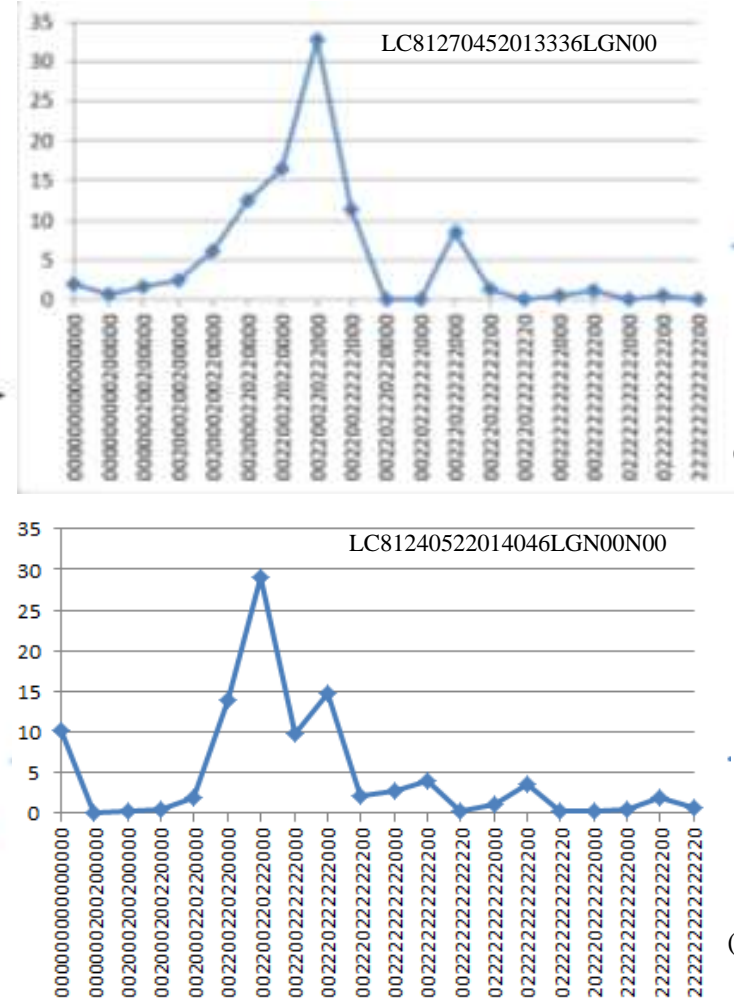

Figure 3. Layout of SRC modulation pattern histogram (a), SRC modulation histograms of Landsat 8 OLI image: LC81270452013336LGN00 (b), LC81240512014030LGN00(c), LC81240522014046LGN00 (d) 
Nguyen Dinh Duong et al./Vietnam Journal of Earth Sciences 36 (2014)

Using this formula to encode SRC in Figure 2, we can obtain the following results:

Water pixel vector $(9.2,6.8,4.8,3.0,0.8$, 0.4),SRC code: 000000000000000

Vegetation pixel vector $(8.6,7.6,5.4,28.0$, 15.4, 7.7),SRC code: 002200222222000

Barren land pixel vector $(11.4,12.8,16.6,22.0$, 30.8, 22.8), SRC code: 222222222222220

Cloud pixel vector $(48.8,50.6,54.6,65.6,55.4$, 44.6), SRC code: 222202220220000

Algorithm forSRCmodulation encoding is given bellow:

Declare integer* 1 array modulation with 15 elements

Set array modulation to 1

While not the last pixel vector of the image

Set variable $\mathrm{k}=0$

\{

$$
\text { for }(\mathrm{i}=0 ; \mathrm{i}<5 ; \mathrm{i}++)
$$$$
\text { for }(j=i+1 ; j<6 ; j++)
$$

$$
\{
$$

if(pixel vector $[\mathrm{j}]<$ pixel vector[i]) set array modulation $[\mathrm{k}]$ to 0

if(pixel vector [j]>pixel vector[i]) set array modulation[k] to 2

$$
\text { \} }
$$

$$
\}
$$

$$
\mathrm{k}++
$$

Because the modulation pattern of SRC is composed of 15 digits, which exceed any integer value range in 32-bit programming environment so it is recommended to use either string variable or integral array to keep value of patterns or better to switch to 64-bit programming environment. Theoretically, there are three power 15 or $14,348,907$ possible modulation patterns for dataset with six bands. However, in practice the number of modulation patterns is fewer and depends on local land cover condition.

According to spectral reflectance characteristics of land cover, we can expect that modulation pattern 000000000000000 represents clear water because the reflectance of water reduces with increase of wavelength. Modulation pattern 222222222222222 corresponds to dry bare land due to spectral reflectivity growth as wavelength increases. Modulation pattern 002200222222000 is typical for green vegetation (forest) because of slight high reflectance in green, low reflectance in red band and peak in near infrared band. This characteristic can be recognized when modulation pattern is filled by 2 in the middle part of the pattern. After conversion of pixel vectors to modulations patterns, we can construct histogram to show their frequency. The modulation patterns are sorted from code 000000000000000 to 222222222222222 or by other words, from water to bare land region. Shape of SRC modulation pattern histogram varies from area to area depending on land cover conditions.

Figure 2 shows histogram template and histograms of modulation patterns for three Landsat OLI image used in this study. By sorting the SRC modulation patterns from water to barren land region, left site of the histogram is water; near center part is devoted to vegetation and right part to a barren land. Histograms of major SRC modulation patterns of the three Landsat OLI images provide information not only about frequency of modulation patterns in each image but also about general land cover situation in each image. By studying the histogram, we can estimate the percentage of water, vegetation and barren land in each image.

\section{Results}

To understand the relation between SRCmodulation pattern and land cover types we decompose the OLI image data to component image with pixel vector of similar spectral signature or the same SRC modulation pattern.

There are 559 SRC modulation patterns in the scene LC81270452013336LGN00. Pattern 002200220222000 has the highest frequency with value of $13,570,460$, which is equivalent to 32.69 $\%$ of pixels in the scene. There are many SRCmodulation patterns with very small 
Vietnam Journal of Earth Sciences 36 (2014) 480-488

frequency. Table 2 show distribution of SRC modulation patterns for all three images. Only SRC modulation patterns, which have high enough frequency values, will be taken into account. For the scene LC81240512014030LGN00, there are 725 SRC modulation patterns in total. However, only 26 SRC modulation patterns dominate with
$40,976,765$ pixels corresponding to $98.57 \%$ pixels in the image. We can achieve a similar result with the scene LC81240522014046LGN00. There are 674 SRC modulation patterns in total in this image, among them 25 SRC modulation patterns occupy $98.77 \%$ of total pixels equivalent to 41074629 pixels.

Table 2.List of dominating SRC modulation patterns in the three Landsat 8 OLIimages

\begin{tabular}{|c|c|c|c|c|}
\hline Landsat 8 OLI scene & SRC modulation patterns & $\begin{array}{l}\text { Number of } \\
\text { pixels }\end{array}$ & Percentage & Land cover type \\
\hline \multirow{19}{*}{ LC81270452013336LGN00 } & 000000000000000 & 847910 & 2.04 & Water, wetlands \\
\hline & 000000000200000 & 267380 & 0.64 & Water, wetlands \\
\hline & 000000200200000 & 680690 & 1.64 & $\begin{array}{l}\text { Cloud shadow, shadow, thin cloud, } \\
\text { wetlands }\end{array}$ \\
\hline & 002000200200000 & 1042610 & 2.51 & Wetlands, cloud shadow, thin cloud \\
\hline & 002000200220000 & 2513475 & 6.05 & Forest, thin cloud \\
\hline & 002000220220000 & 5215137 & 12.56 & Forest, thin cloud, developed, grassland \\
\hline & 002200220220000 & 6863976 & 16.53 & Forest, barren land, grassland, thin cloud \\
\hline & 002200220222000 & 13570460 & 32.69 & Forest, shrub land, grassland \\
\hline & 002200222222000 & 4723985 & 11.38 & $\begin{array}{l}\text { Forest, barren land, grassland, developed } \\
\text { land }\end{array}$ \\
\hline & 002202220220000 & 60682 & 0.15 & Thin cloud \\
\hline & 002202222222000 & 37069 & 0.09 & Barren land \\
\hline & 002220222222000 & 3517290 & 8.47 & Forest \\
\hline & 002220222222200 & 530880 & 1.28 & Barren land, developed land \\
\hline & 002220222222220 & 54708 & 0.13 & Developed land \\
\hline & 002222222222000 & 257839 & 0.62 & Barren land \\
\hline & 002222222222200 & 486056 & 1.17 & Barren land \\
\hline & 022222222222000 & 42855 & 0.10 & Thin cloud, barren land \\
\hline & 022222222222200 & 221814 & 0.53 & Barren land, developed land \\
\hline & 222222222222200 & 41949 & 0.10 & Barren land, developed land \\
\hline Total & & 40976765 & 98.70 & \\
\hline \multirow{19}{*}{ LC81240512014030LGN00 } & 000000000000000 & 241070 & 0.58 & Water \\
\hline & 000000200200000 & 63458 & 0.15 & wetlands \\
\hline & 002000200200000 & 173716 & 0.42 & wetlands \\
\hline & 002000200220000 & 278102 & 0.67 & Wetlands, Cloud shadow \\
\hline & 002000220220000 & 1068798 & 2.57 & Shadow, Forest \\
\hline & 002200220220000 & 2085190 & 5.01 & Forest, Shadow \\
\hline & 002200220222000 & 10979782 & 26.40 & Forest \\
\hline & 002200222222000 & 4414831 & 10.62 & Forest, Shrub land \\
\hline & 002202222222000 & 65173 & 0.16 & Developed land, Cloud \\
\hline & 002220222222000 & 8318551 & 20.00 & Shrub land, Grassland \\
\hline & 002220222222200 & 901276 & 2.17 & Barren land \\
\hline & 002220222222220 & 140485 & 0.34 & Barren land \\
\hline & 002222222222000 & 2365639 & 5.69 & Barren land, Shrubland, Thick cloud \\
\hline & 002222222222200 & 3092712 & 7.44 & Barren land, Grassland \\
\hline & 002222222222220 & 1234670 & 2.97 & Barren land, Developed land \\
\hline & 022222222222000 & 980222 & 2.36 & Barren land, Developed land, Grassland \\
\hline & 022222222222200 & 2691608 & 6.47 & Barren land, Developed land \\
\hline & 022222222222220 & 483761 & 1.16 & Barren land, Developed land \\
\hline & 200000000000000 & 160586 & 0.39 & Water \\
\hline
\end{tabular}


Nguyen Dinh Duong et al./Vietnam Journal of Earth Sciences 36 (2014)

\begin{tabular}{|c|c|c|c|c|}
\hline Landsat 8 OLI scene & SRC modulation patterns & $\begin{array}{l}\text { Number of } \\
\text { pixels }\end{array}$ & Percentage & Land cover type \\
\hline & 202200220220000 & 35215 & 0.08 & Grassland, Developed land \\
\hline & 202200220222000 & 69882 & 0.17 & Grassland, Thin cloud \\
\hline & 202220222222000 & 179328 & 0.43 & Shrub land, Grassland, Forest \\
\hline & 222222222222000 & 197946 & 0.48 & $\begin{array}{l}\text { Grassland, Shrub land, Barren land, } \\
\text { cloud }\end{array}$ \\
\hline & 222222222222200 & 652262 & 1.57 & Barren land \\
\hline & 222222222222220 & 200366 & 0.48 & Barren land \\
\hline & 000000000000000 & 241070 & 0.58 & Water \\
\hline Total & & 41074629 & 98.77 & \\
\hline \multirow{20}{*}{ LC81240522014046LGN00 } & 000000000000000 & 4247018 & 10.21 & Water \\
\hline & 000000200200000 & 60893 & 0.15 & Water, cloud shadow \\
\hline & 002000200200000 & 133033 & 0.32 & Wetlands, cloud shadow \\
\hline & 002000200220000 & 210399 & 0.51 & Wetlands, cloud shadow \\
\hline & 002000220220000 & 805420 & 1.94 & Forest, grassland, wetlands, thin cloud \\
\hline & 002200220220000 & 5770519 & 13.88 & Forest, grassland, Wetlands, thin cloud \\
\hline & 002200220222000 & 12104829 & 29.11 & Forest, shrub land \\
\hline & 002200222222000 & 4095455 & 9.85 & Shrub land, thin cloud, Developed land \\
\hline & 002220222222000 & 6128662 & 14.74 & Shrub land, Developed land, Thin cloud \\
\hline & 002220222222200 & 866822 & 2.08 & Barren land, Developed land, \\
\hline & 002222222222000 & 1182658 & 2.84 & Barren land, Developed land, Cloud \\
\hline & 002222222222200 & 1631302 & 3.92 & Barren land, Developed land \\
\hline & 002222222222220 & 119485 & 0.29 & Developed land, Barren land \\
\hline & 022222222222000 & 466472 & 1.12 & Barren land, Developed land, Cloud \\
\hline & 022222222222200 & 1456403 & 3.50 & Barren land \\
\hline & 022222222222220 & 145827 & 0.35 & Barren land \\
\hline & 202220222222000 & 91062 & 0.22 & Shrub land \\
\hline & 222222222222000 & 190583 & 0.46 & Developed land, Cloud, Barren land \\
\hline & 222222222222200 & 782381 & 1.88 & Barren land \\
\hline & 222222222222220 & 267367 & 0.64 & Barren land \\
\hline Total & & 40756590 & 98.01 & \\
\hline
\end{tabular}

Land cover types associated with each SRC modulation pattern in Table 2 are the result of visual interpretation. For illustration, we select some SRC modulation patterns for each Landsat 8
OLI scenes. Figure 4 show SRC modulation patterns image for a window around Hanoi City extracted from the scene LC81270452013336LGN00.

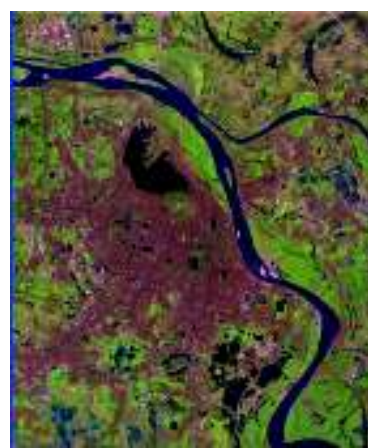

(a)

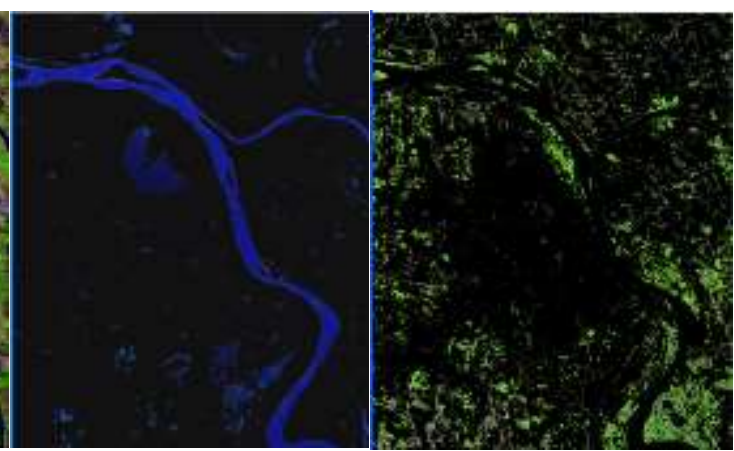

(b) (c)

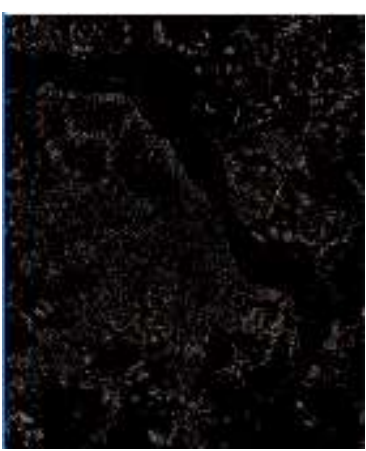

(d)

Figure 4. (a) short wave infrared color composite RGB: 5, 4, 3; (b) pattern 000000000000000 - water;

(b) pattern 002200220220000 - vegetation; (d) pattern 002220222222200-barren land, build up 
Vietnam Journal of Earth Sciences 36 (2014) 480-488

This image window covers Hanoi City and surrounding areas. There are developed land, paddy field, rivers and lakes. Figure 4 (a) shows short wave infrared color composite by assigning band 5 to red, 4 to green and 3 to blue. In this color composite, water appears in bluish, vegetation in the greenish tint and barren land in different grades of magenta. We present spectral pattern 000000000000000 in Figure 4 (b). Here we can observe that almost all water bodies including river, lakes, ponds and wetland. Spectral pattern 002200220220000 is dominated by green vegetation and in this image window it is mainly agricultural cultivation. The pattern 002220222222200 involves barren land and built up, which is closely related to the urban area of Hanoi City.
Figure 5 displays an extract from scene LC81240512014030LGN00 that covers an area of highland Tay Nguyen. Land cover of this area features evergreen vegetation on the mountain, deciduous forest and shrub on plateaus land and paddy field around rivers and streams. Again, Figure 5 (a) is short wave infrared color composite RGB: 5, 4, 3. We can distinguish evergreen vegetation by greenish color, water by bluish tone and brown vegetation by magenta in different shades. Image of pattern 000000000000000 in Figure 5 (b) shows large water bodies of the image window. Figure 5 (c) is an image of pattern 002200220222000 that gives the distribution of green vegetation. Barren land and brown shrub (shrub during fall leaf season) is the main content of Figure $5(d)$, which is the image of pattern 022222222222200 .

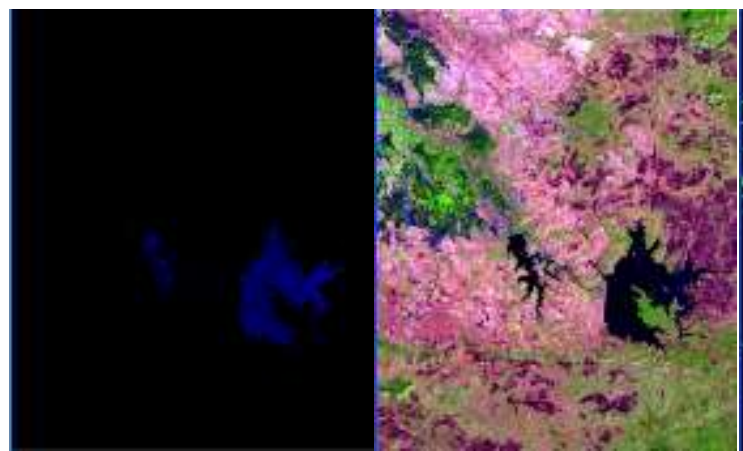

(a)

(b)

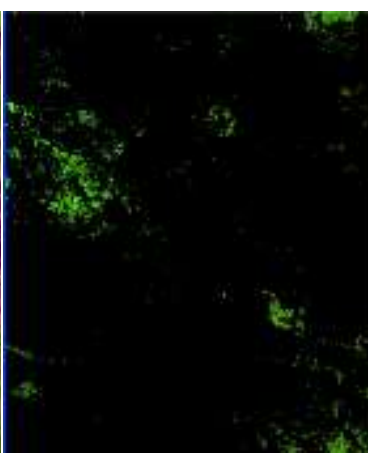

(c)

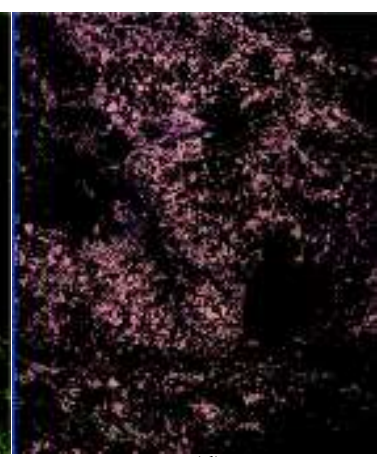

(d)

Figure 5.(a) short wave infrared color composite RGB: 5, 4, 3; (b) pattern 000000000000000 - water;(c) pattern 002200220222000 green vegetation; (d) pattern 02222222222200 - barren land, brownshrub

Figure 6 shows a portion of the scene LC81240522014046LGN00. Evergreen forest dominates the landscape of this area. Images of patterns 000000000000000, 002200220222000 and 022222222222200 are presented in Figure
6 (a), 6 (b)and 6 (c) respectively. Evergreen forest and water body are closely related with patterns 002200220222000 and 000000000000000 . Unlike Figure 5 (d), the pattern 022222222222200 in Figure 6 (d) shows bush and grassland.

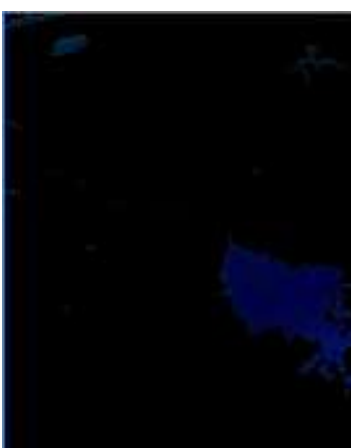

(a)

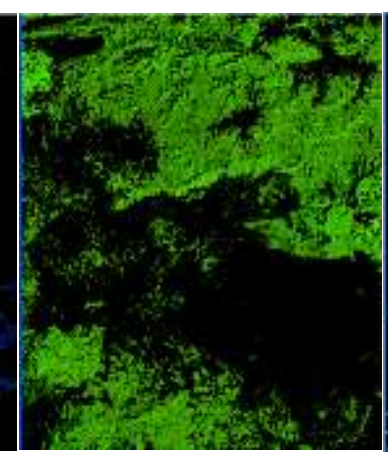

(b)

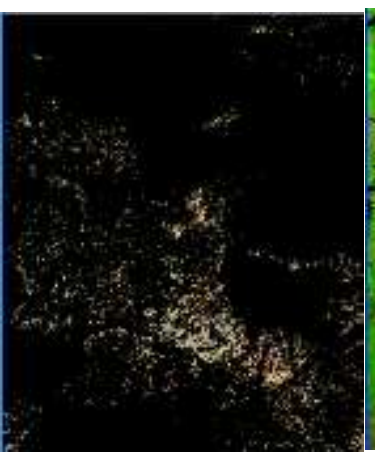

(c)

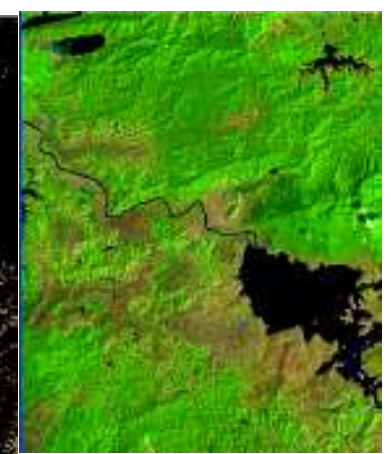

(d)

Figure 6. (a) short wave infrared color composite RGB: 5, 4, 3; (b) pattern 000000000000000 - water; (c) pattern 002200220222000 - forest; (d) pattern 022222222222200-bush and grassland 
Nguyen Dinh Duong et al./Vietnam Journal of Earth Sciences 36 (2014)

\section{Discussion and conclusion}

In this research, we grouped image pixels according to their SRC modulation patterns to single component image and tried to explain meaning of each modulation pattern by visual interpretation. There are hundreds of SRC modulation patterns in each Landsat 8 OLI scene. Frequency of patterns varies from some millions to one pixel in one image scene. However, number of patterns with large enough frequency is quite small and about some tens only. There are 40,976,765 pixels in the scene LC81270452013336LGN00 distributed in 559 SRC modulation patterns, but only 19 patterns with the highest frequency comprise $98.7 \%$ of total pixels. The scene LC81240512014030LGN00 has 41,591,459 pixels spreading over 725 SRC modulation patterns. In this scene, $98.6 \%$ of total pixels equivalent to 40,996,002 pixels are allocated in only 26 SRC modulation patterns. Similarly, number of significant SRC modulation patterns in the scene LC81240522014046LGN00 is 20 of 585. These 20 SRC modulation patterns contain 40756590 pixels equivalent to $98.0 \%$ of total pixels in this scene.

We found out by visual interpretation that each SRC modulation pattern is closely related to very limited number of land cover types. Some patterns like 00000000000000, 002200220222000, 22222222222220 are interpreted consistently as water, green vegetation and barren land. The other patterns are a mixturebetween water and vegetation or vegetation and barren land. Meaning ofthosepatterns is stable in all three Landsat OLI scenes. To confirm interpretation of SRC modulation pattern we use GPS field photos, which we took during the field trip in 2013. Figure 7 shows field trip and location wherethe GPS field photos were acquired. Figure 8 demonstrates examples of land cover and SRC modulation patterns in relation to ground GPS field photos.

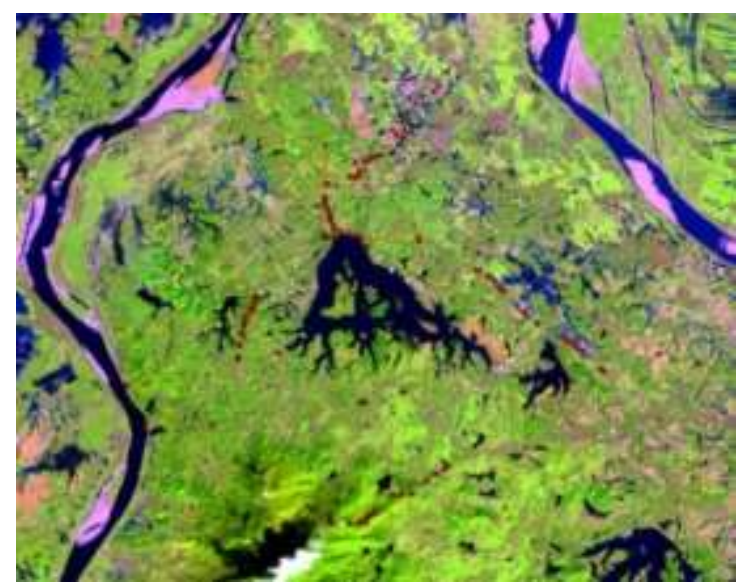

Figure 7. Field trip with location of GPS fieldphotos in Dong Mo area
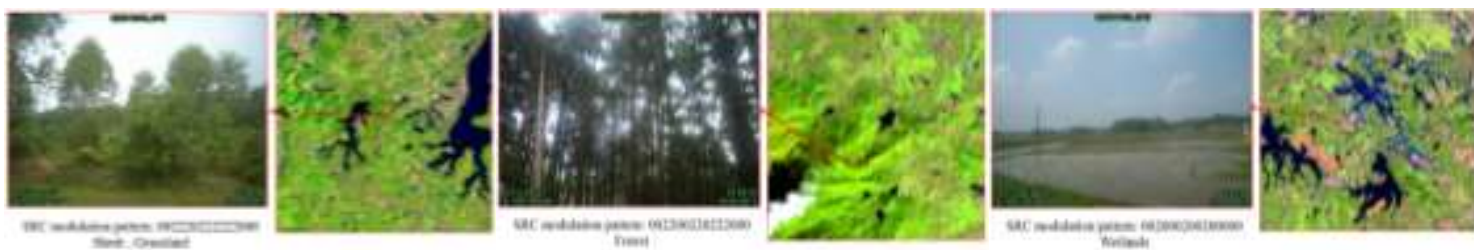

Figure 8. An example of land cover and SRC modulation patterns in relation to ground GPS field photos

In conclusion, the paper demonstrated a new approach to understanding spectral signatures of Landsat 8 OLI image. By grouping of pixel vectors to limited SRC modulation patterns, we can establish a relation between each pixel to a limited number of land cover types, which helps to easier and better land cover interpretation. We have found out interesting observation that meaning of SRC modulation patterns is stable in different Landsat 8 OLI scenes over different geographic regions. This finding could lay the foundation for the development of a new method for land cover classification.

\section{Acknowledgement}

This research is funded by Vietnam National Foundation for Science and Technology Development (NAFOSTED) under grant number 105.08-2014.14. 


\section{Vietnam Journal of Earth Sciences 36 (2014) 480-488}

\section{Reference}

Canty, M.J., Nielsen, A.A., Schmidt, M., 2004: Automatic radiometric normalization of multitemporal satellite imagery. Remote Sensing of Environment 91.3, 441-451.

Duong, N. D.,1997: Graphical analysis of spectral reflectance curve.In Proceedings of the 18th Asian Conference on Remote Sensing, Oct 1997, pp. 20-24.

Laborte, Alice G., Maunahan, A.A., Hijmans, R.J., 2010: Spectral signature generalization and expansion can improve the accuracy of satellite image classification. PloS one 5.5, e10516.

Olthof, I., Butson, C., Fraser, R., 2005: Signature extension through space for northern landcover classification: A comparison of radiometric correction methods. Remote Sensing of Environment 95.3, 290-302.

Ruby, J.G., Fischer, R.L., 2002: Spectral signatures database for remote sensing applications. International Symposium onOptical Science and TechnologyInternationalSociety for Optics and Photonics, 2002.

Shaw, G.A., Burke, H-h.K, 2003: Spectral imaging for remote sensing". Lincoln Laboratory Journal 14.1, 3-28.

Thenkabail, P., et al., 2007: Spectral matching techniques to determine historical land-use/land-cover (LULC) and irrigated areas using time-series 0.1-degree AVHRR Pathfinder datasets. Photogrammetric Engineering \& Remote Sensing73.10, 1029-1040. 